Document downloaded from:

http://hdl.handle.net/10251/176355

This paper must be cited as:

López Alfonso, S.; López Pellicer, M. (2020). Weak Sequential Convergence in Bounded Finitely Additive Measures. Vietnam Journal of Mathematics. 48(2):379-389. https://doi.org/10.1007/s10013-020-00387-2

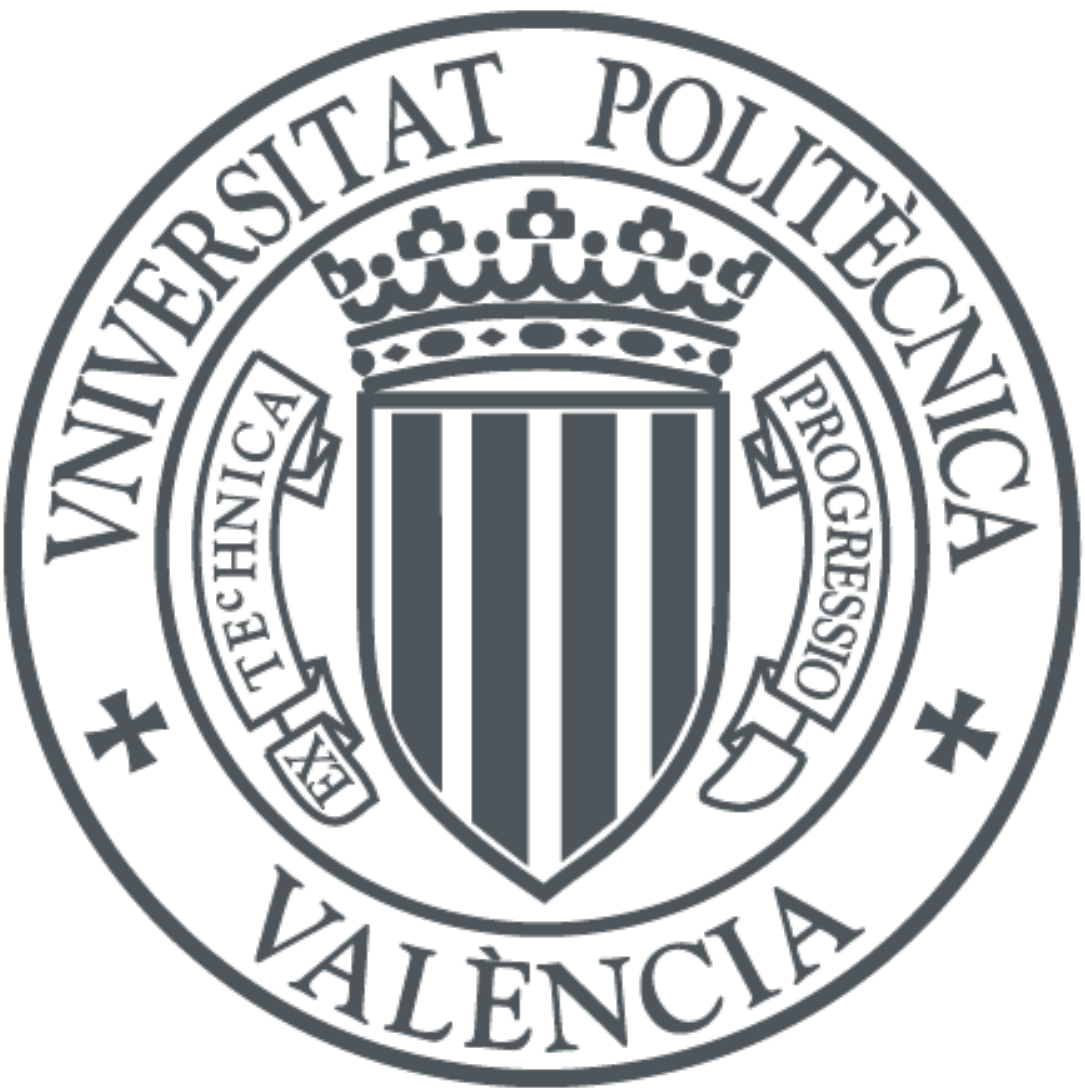

The final publication is available at

https://doi.org/10.1007/s10013-020-00387-2

Copyright Springer

Additional Information 


\section{Weak Sequential Convergence in Bounded Finitely Additive Measures}

\section{Salvador López-Alfonso \& Manuel López-Pellicer}

\section{Vietnam Journal of Mathematics}

ISSN 2305-221X

Volume 48

Number 2

Vietnam J. Math. (2020) 48:379-389

DOI 10.1007/s10013-020-00387-2 
Your article is protected by copyright and all rights are held exclusively by Vietnam Academy of Science and Technology (VAST) and Springer Nature Singapore Pte Ltd.. This e-offprint is for personal use only and shall not be self-archived in electronic repositories. If you wish to self-archive your article, please use the accepted manuscript version for posting on your own website. You may further deposit the accepted manuscript version in any repository, provided it is only made publicly available 12 months after official publication or later and provided acknowledgement is given to the original source of publication and a link is inserted to the published article on Springer's website. The link must be accompanied by the following text: "The final publication is available at link.springer.com". 


\title{
Weak Sequential Convergence in Bounded Finitely Additive Measures
}

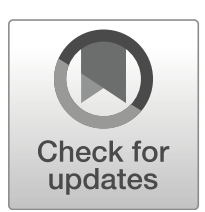

\author{
Salvador López-Alfonso ${ }^{1}$ - Manuel López-Pellicer² ${ }^{2}$
}

Received: 8 August 2019 / Accepted: 11 October 2019 / Published online: 2 March 2020

(C) Vietnam Academy of Science and Technology (VAST) and Springer Nature Singapore Pte Ltd. 2020

\begin{abstract}
It is well known that a $\sigma$-algebra $\Sigma$ of subsets of a set $\Omega$ verifies both Nikodým property and property $(G)$ for the Banach space $b a(\Sigma)$ of bounded finitely additive measures defined in $\Sigma$. A classic result of Valdivia stating that if a $\sigma$-algebra $\Sigma$ is covered by an increasing sequence $\left(\Sigma_{n}: n \in \mathbb{N}\right)$ of subsets, there is $p \in \mathbb{N}$ such that $\Sigma_{p}$ is a Nikodým set for $b a(\Sigma)$ was complemented in Ferrando et al. (2020) proving that there exists $p \in \mathbb{N}$ such that $\Sigma_{p}$ is both a Nikodým and a Grothendieck set for $b a(\Sigma)$. Valdivia result was the first step to get that if $\left(\Sigma_{\sigma}: \sigma \in \mathbb{N}^{<\infty}\right)$ is a web in $\Sigma$ there exists a chain $\left(\sigma_{n}: n \in \mathbb{N}\right)$ in $\mathbb{N}<\infty$ such that each $\Sigma_{\sigma_{n}}, n \in \mathbb{N}$, is a Nikodým set for $b a(\Sigma)$. In this paper, we develop several properties in Banach spaces that enables us to complement the preceding web result extending the main result in Ferrando et al. (2020) proving that for each web $\left(\Sigma_{\sigma}: \sigma \in \mathbb{N}^{<\infty}\right)$ in a $\sigma$-algebra $\Sigma$ there exists a chain $\left(\sigma_{n}: n \in \mathbb{N}\right)$ in $\mathbb{N}<\infty$ such that each $\Sigma_{\sigma_{n}}, n \in \mathbb{N}$, is both a Nikodým and a Grothendieck set for $b a(\Sigma)$. As an application we extend some results of classic Banach space theory.
\end{abstract}

Keywords Algebras and $\sigma$-algebras of sets · Bounded finitely additive measures · Grothendieck · Nikodým and Rainwater sets · Pointwise and weak sequential convergence $\cdot$ Web properties

Mathematics Subject Classification (2010) 28A33 · 46B25

To Professor Marco Antonio López at the occasion of his 70th birthday.

Manuel López-Pellicer mlopezpe@mat.upv.es

Salvador López-Alfonso salloal@csa.upv.es

1 Depto. de Construcciones Arquitectónicas, Universitat Politècnica de València, E-46022 Valencia, Spain

2 Depto. de Matemática Aplicada and IUMPA, Universitat Politècnica de València, E-46022 Valencia, Spain 


\section{Introduction}

Let $\mathcal{A}$ be an algebra of subsets of a nonempty set $\Omega, \chi_{A}$ be the characteristic function of the set $A \in \mathcal{A}$ and let $\ell_{0}^{\infty}(\mathcal{A})=\operatorname{span}\left\{\chi_{A}: A \in \mathcal{A}\right\}$ denote the linear space of all $\mathbb{K}$-valued $\mathcal{A}$ simple functions, $\mathbb{K}$ being the scalar field of real or complex numbers. For each $f \in \ell_{0}^{\infty}(\mathcal{A})$ there are pairwise disjoint sets $A_{1}, \ldots, A_{m} \in \mathcal{A}$ and nonzero $a_{1}, \ldots, a_{m} \in \mathbb{K}$, with $a_{i} \neq a_{j}$ if $i \neq j$, such that $f=\sum_{i=1}^{m} a_{i} \chi_{A_{i}}$, with $f=\chi_{\emptyset}$ if $f=0$. We shall assume $\ell_{0}^{\infty}(\mathcal{A})$ is equipped with the norm $\|f\|=\sup \{|f(\omega)|: \omega \in \Omega\}$. The completion of the normed space $\left(\ell_{0}^{\infty}(\mathcal{A}),\|\cdot\|\right)$ is the Banach space $\ell_{\infty}(\mathcal{A})$ of all bounded $\mathcal{A}$-measurable functions.

The dual of $\ell_{0}^{\infty}(\mathcal{A})$ is the Banach space $b a(\mathcal{A})$ of bounded finitely-additive measures on $\mathcal{A}$, which we shall assume equipped with the dual norm of $\|\cdot\|$, named variation norm and defined by

$$
|\mu|=\sup \sum_{i=1}^{n}\left|\mu\left(E_{i}\right)\right|,
$$

where the supremum is taken over all finite pairwise disjoint members of $\mathcal{A}$. We shall also consider the Banach space $b a(\mathcal{A})^{*}$ equipped with the bidual norm, also denoted by $\|\cdot\|$.

Since the Banach space $\ell_{\infty}(\mathcal{A})$ is linearly isometric to a subspace of $b a(\mathcal{A})^{*}$ then each characteristic function $\chi_{A} \in \ell_{0}^{\infty}(\mathcal{A})$, with $A \in \mathcal{A}$, can be considered as a bounded linear functional on $b a(\mathcal{A})$ defined by $\left\langle\chi_{A}, \mu\right\rangle=\mu(A)$ and $\left\{\chi_{A}: A \in \mathcal{A}\right\} \subseteq S_{b a(\mathcal{A})^{*} \text {, where }}$ $S_{b a(\mathcal{A})^{*}}$ stands for the unit sphere of $b a(\mathcal{A})^{*}$.

Let $\mathbb{N}^{<\infty}$ be the family of all finite sequences of natural numbers and the empty sequence $\emptyset$. We denote by $\sigma \frown n$ the concatenation of a $\sigma \in \mathbb{N}<\infty$ followed by a natural number $n$. A sequence $\left(\sigma_{n}: n \in \mathbb{N}\right)$ in $\mathbb{N}<\infty$ is a chain if for each $n \in \mathbb{N}$ there exists $m_{n+1}$ such that $\sigma_{n+1}=\sigma_{n} \frown m_{n+1}$. A sequence $\left(A_{\sigma}: \sigma \in \mathbb{N}^{<\infty}\right)$ of subsets of $A$ is a web in $A$ if $A=A_{\emptyset}$ and for each $\sigma \in \mathbb{N}^{<\infty}$ the sequence $\left(A_{\sigma \frown n}: n \in \mathbb{N}\right)$ is an increasing covering of $A_{\sigma}$.

The following definitions were introduced by Schachermayer [20], Valdivia [25], LópezAlfonso, Mas and Moll [13], Kakol and López-Pellicer [12] and Ferrando, López-Alfonso and López-Pellicer [7].

Definition 1 A subfamily $\Delta$ of an algebra of sets $\mathcal{A}$ is called a $\operatorname{Nikodým~set~for~} b a(\mathcal{A})$ if each $\Delta$-pointwise bounded subset $\left\{\mu_{\alpha}: \alpha \in \Lambda\right\}$ of $b a(\mathcal{A})$ is bounded in $b a(\mathcal{A})$, i.e.,

$$
\sup _{\alpha \in \Lambda}\left|\mu_{\alpha}(A)\right|<\infty \quad \text { for each } A \in \Delta \Rightarrow \sup _{\alpha \in \Lambda}\left|\mu_{\alpha}\right|<\infty \text {. }
$$

Definition 2 A subfamily $\Delta$ of an algebra of sets $\mathcal{A}$ is called a strong Nikodým set for $b a(\mathcal{A})$ if for each increasing covering $\left(\Delta_{n}: n \in \mathbb{N}\right)$ of $\Delta$ there exists $n_{0} \in \mathbb{N}$ for which $\Delta_{n_{0}}$ is a Nikodým set for $b a(\mathcal{A})$.

Definition 3 A subfamily $\Delta$ of an algebra of sets $\mathcal{A}$ is called a web Nikodým set for $b a(\mathcal{A})$ if for each web $\left(\Delta_{\sigma}: \sigma \in \mathbb{N}^{<\infty}\right)$ in $\Delta$ there exists a chain $\left(\sigma_{n}: n \in \mathbb{N}\right)$ in $\mathbb{N}^{<\infty}$ such that each $\Delta_{\sigma_{n}}, n \in \mathbb{N}$, is a Nikodým set for $b a(\mathcal{A})$.

An algebra of sets $\mathcal{A}$ is said to have the Nikodým property, the strong Nikodým property or the web Nikodým property if the family $\mathcal{A}$ is a Nikodým set, a strong Nikodým set or a web Nikodým set for $b a(\mathcal{A})$, respectively.

It is well known that every countable algebra lacks Nikodým property and that the classical Nikodým-Grothendieck boundedness theorem states that each $\sigma$-algebra of sets $\Sigma$ 
has the Nikodým property. In [24, Theorem 1], using methods of barrelled spaces, Valdivia showed each $\sigma$-algebra of sets $\Sigma$ has the strong Nikodým property. This result was later strengthened by López-Pellicer [15] by showing that each $\sigma$-algebra of sets $\Sigma$ even has the web Nikodým property. The book [9] collects together these results as well as previous research on Nikodým sets and Nikoddým's property until 1995. In [22] is considered the Nikodým property in the Sacks model.

An algebra $\mathcal{A}$ is said to have property $(G)$ if $\ell_{\infty}(\mathcal{A})$ is a Grothendieck space, i.e., if each weak* convergent sequence in $b a(\mathcal{A})$ is weak convergent, i.e., for each sequence $\left(\mu_{n}\right)_{n}$ in $b a(\mathcal{A})$ such that $\lim _{n} \mu_{n}(f)=\mu(f)$, for each $f \in \ell_{\infty}(\mathcal{A})$, we have that $\lim _{n} \varphi\left(\mu_{n}\right)=\varphi(\mu)$, for each $\varphi \in b a(\mathcal{A})^{*}$. The classical property that every $\sigma$-algebra has property $(G)$ is also due to Grothendieck. In [20, Propositions 3.2 and 3.3] it is proved that the algebra $\mathfrak{J}$ of Jordan subsets of the real interval $[0,1]$ has Nikodým property but fails property $(G)$. The Nikodým part of this result was recently strengthened in [14, Theorem 1] stating that the algebra of Jordan measurable subsets of the $n$-dimensional cube has the web Nikodým property. We shall use in Lemma 2 the characterization of property $(G)$ given in Theorem 1. Let us recall that a sequence $\left\{\mu_{n}\right\}_{n=1}^{\infty}$ in $b a(\mathcal{A})$ is uniformly exhaustive on $\mathcal{A}$ if for each sequence $\left\{A_{i}: i \in \mathbb{N}\right\}$ of pairwise disjoint elements of $\mathcal{A}$ it holds that $\lim _{k \rightarrow \infty} \sup _{n \in \mathbb{N}}\left|\mu_{n}\left(A_{k}\right)\right|=0$.

Theorem 1 ([20, 2.3. Definition]) An algebra of sets $\mathcal{A}$ has property $(G)$ if and only if every bounded sequence $\left(\mu_{n}\right)_{n=1}^{\infty}$ in ba $(\mathcal{A})$ which converges pointwise on $\mathcal{A}$ is uniformly exhaustive.

An algebra $\mathcal{A}$ is said to have property $(V H S)$ if every sequence $\left(\mu_{n}\right)_{n=1}^{\infty}$ in $b a(\mathcal{A})$ which converges pointwise on $\mathcal{A}$ is uniformly exhaustive. It should be mentioned that $\mathcal{A}$ has property $(V H S)$ if and only if it has both Nikodým's property and property $(G)$, where the proof of the non-trivial implication can be found in [2] (see also [10, Theorem 4.2]).

The following definitions are motivated by property $(G)$, by the method used in definitions of the strong and web Nikodým sets and by some natural properties we are going to obtain in Corollary 3 and later in Theorem 3.

Definition 4 A subfamily $\Delta$ of an algebra of sets $\mathcal{A}$ will be called a Grothendieck set for $b a(\mathcal{A})$ if each sequence $(\mu)_{n n=1}^{\infty}$ in $b a(\mathcal{A})$ which is pointwise convergent on $\Delta$ is weak convergent in $b a(\mathcal{A})$, i.e., if there is $\mu \in b a(\mathcal{A})$ such that $\mu_{n}(A) \rightarrow \mu(A)$ for every $A \in \Delta$ then $\varphi\left(\mu_{n}\right) \rightarrow \varphi(\mu)$, for each $\varphi \in b a(\mathcal{A})^{*}$.

Clearly if $\Delta$ is a Grothendieck set for $b a(\mathcal{A})$ then $\mathcal{A}$ is a Grothendieck set for $b a(\mathcal{A})$ and this obviously implies that the algebra $\mathcal{A}$ has property $(G)$. The converse statements fail in general, as follows from [23] where under $\mathrm{CH}$ Talagrand gives an algebra of sets $\mathcal{A}$ with the property $(G)$ for which there exists a sequence $(\mu)_{n=1}^{\infty}$ in $b a(\mathcal{A})$ such that $\mu_{n}(A) \rightarrow 0$ for all $A \in \mathcal{A}$, but $\left\|\mu_{n}\right\| \rightarrow \infty$, hence the algebra $\mathcal{A}$ lacks the Nikodým property. Moreover $\mathcal{A}$ is not a Grothendieck set for $b a(\mathcal{A})$. Otherwise, we would have $\varphi\left(\mu_{n}\right) \rightarrow 0$, for each $\varphi \in b a(\mathcal{A})^{*}$, a contradiction with $\left\|\mu_{n}\right\| \rightarrow \infty$.

Definition 5 A subfamily $\Delta$ of an algebra of sets $\mathcal{A}$ is called a strong Grothendieck set for $b a(\mathcal{A})$ if for each increasing covering $\left(\Delta_{n}: n \in \mathbb{N}\right)$ of $\Delta$ there exists $n_{0} \in \mathbb{N}$ for which $\Delta_{n_{0}}$ is a Grothendieck set for $b a(\mathcal{A})$. 
Definition 6 A subfamily $\Delta$ of an algebra of sets $\mathcal{A}$ is called a web Grothendieck set for $b a(\mathcal{A})$ if for each web $\left(\Delta_{\sigma}: \sigma \in \mathbb{N}^{<\infty}\right)$ in $\Delta$ there exists a chain $\left(\sigma_{n}: n \in \mathbb{N}\right)$ in $\mathbb{N}<\infty$ such that each $\Delta_{\sigma_{n}}, n \in \mathbb{N}$, is a Grothendieck set for $b a(\mathcal{A})$.

\section{Uniform Bounded Deciding Sets (ubd Sets)}

The next definition appear in [5] and it has been used, for instance, in [16-18], among others.

Definition 7 (Fernández, Hui, and Shpiro, 1989) A subset $C$ of a normed space $E$ is an uniform bounded deciding set ( $u b d$ set, in brief) for $E^{*}$ if each subset $M$ of $E^{*}$ which is pointwise bounded on $C$ is norm bounded, i.e.,

$$
\sup _{f \in M}|f(x)|<\infty, \quad \forall x \in C \quad \Longrightarrow \quad \sup _{f \in M}\|f\|=\sup _{f \in M, x \in B_{E}(0,1)}|f(x)|<\infty .
$$

As we may suppose that $0 \notin C$, we assume that $0 \notin C$. Then the obvious equivalence

$$
C \text { is } u b d \quad \Leftrightarrow \quad\left\{\|x\|^{-1} x: x \in C \backslash\{0\}\right\} \text { is } u b d
$$

enables us to consider that each $u b d$ set for $E^{*}$ is a subset of the unit sphere $S_{E}$ of $E$. The Banach-Steinhaus theorem says that for a Banach space $(E,\|\cdot\|)$ the closed unit ball $B_{E}$ and the unit sphere $S_{E}$ are $u b d$ sets for $E^{*}$.

Fonf proved in [11] that the set of exposed points of the unit sphere of a reflexive Banach space $E$ is an $u b d$ set for $E^{*}$, hence the set of extreme points of $S_{E}$ is an $u b d$ set for $E^{*}$. The situation for non reflexive Banach spaces is quite different, because it is easy to prove that the sets of extreme points of $S_{l_{\infty}}$ or $S_{L_{\infty}[0,1]}$ are $u b d$ sets for $\left(l_{\infty}\right)^{*}$ or $L_{\infty}[0,1]^{*}$, while it is unknown if there exists proper subsets of the unit spheres $S_{c_{0}}$ or $S_{l_{1}}$ that are $u b d$ sets for $\left(c_{0}\right)^{*}$ or $\left(l_{1}\right)^{*}=l_{\infty}$, respectively.

It is unknown whether the set $C$ of inner functions in the Hardy space on the unit circle $H^{\infty}$ is an $u b d$ set for its dual. This problem was stated in [5, Introduction] and it seems to be a very deep problem.

If $\Delta$ is a subfamily of an algebra of sets $\mathcal{A}$ then $\Delta$ is a Nikodým set for $b a(\mathcal{A})$ if and only if $\left\{\chi_{A}: A \in \Delta\right\}$ is a $u b d$ set for $b a(\mathcal{A})$, since it has been noted that each $A \in \mathcal{A}$ defines a bounded linear functional $\chi_{A}$ on $b a(\mathcal{A})$ determined by $\left\langle\chi_{A}, \mu\right\rangle=\mu(A)$. This observation implies that Nikodým sets may be considered as particular cases of $u b d$ sets and it motivates the following definitions that are natural extensions of strong and web Nikodým sets.

Definition 8 A subset $C$ of a normed space $E$ is a strong $u b d$ set for $E^{*}$ if for each increasing covering $\left(C_{n}: n \in \mathbb{N}\right)$ of $C$ there exists $n_{0} \in \mathbb{N}$ for which $C_{n_{0}}$ is an ubd set for $E^{*}$.

Definition 9 A subset $C$ of a normed space $E$ is a web $u b d$ set for $E^{*}$ if for each web $\left(C_{\sigma}: \sigma \in \mathbb{N}^{<\infty}\right)$ in $C$ there exists a chain $\left(\sigma_{n}: n \in \mathbb{N}\right)$ in $\mathbb{N}<\infty$ such that each $C_{\sigma_{n}}, n \in \mathbb{N}$, is an $u b d$ set for $E^{*}$.

Let $\mathcal{A}$ be an algebra of sets. The next direct lemma summarize the natural relations between classes of Nikodým sets and $u b d$ sets for $b a(\mathcal{A})$. 
Lemma 1 A subfamily $\Delta$ of $\mathcal{A}$ is a (strong, web) Nikodým set for ba $(\mathcal{A})$ if and only if the subset $\left\{\chi_{A}: A \in \Delta\right\}$ of $\ell_{0}^{\infty}(\mathcal{A})$ is a (strong, web) ubd set for ba $(\mathcal{A})$.

Two characterizations of $u b d$ sets are given in Propositions 1 and 2.

Proposition 1 Let $C$ be a subset of a normed space $E . C$ is an ubd set for $E^{*}$ if and only if $\overline{\operatorname{span}\{C\}}=E$ and $C$ is an ubd set for $(\operatorname{span}\{C\})^{*}$.

Proof If $\overline{\operatorname{span}\{C\}} \neq E$ then for each $n$ there exists $f_{n} \in E^{*}$ with $\left\|f_{n}\right\|=n$ and $f_{n}(C)=$ $\{0\}$. So, $C$ would not be an $u b d$ set for $E^{*}$. If $C$ is not $u b d$ set for $(\operatorname{span}\{C\})^{*}$ then $C$ is not $u b d$ set for $E^{*}$. The converse follows from the observation that if $\overline{\operatorname{span}\{C\}}=E$ then the duals $(\operatorname{span}\{C\})^{*}$ and $E^{*}$ are linearly norm isometric.

To get a functional description of $u b d$ sets let us remind that the absolute polar $C^{\circ}$ of a subset $C$ of a normed space $E$ is

$$
C^{\circ}=\left\{x^{*} \in E^{*}:\left|x^{*}(x)\right| \leq 1, x \in C\right\},
$$

and the bipolar of $C$ is $C^{\circ \circ}=\left\{x \in E:\left|x\left(x^{*}\right)\right| \leq 1, x^{*} \in C^{\circ}\right\}$. The bipolar $C^{\circ \circ}$ of $C$ is the closed absolutely convex cover of $C$.

Definition 10 A subset $C$ of a normed space $(E,\|\cdot\|)$ is norming if the Minkowski functional of its bipolar $C^{\circ \circ}$ is a norm in $E$ equivalent to the original norm $\|\cdot\|$. The set $C$ is strong norming if each increasing covering $\left(C_{m}\right)_{m}$ of $C$ contains a norming set $C_{n}$.

Therefore $C$ is norming if there exists $0<r<R$ such that $B(0, r) \subset C^{\circ \circ} \subset B(0, R)$, with $B(0, R)=\{x \in E:\|x\| \leq R\}$. If $E \neq\{0\}$ then $C^{\circ \circ}=\{x: x \in C \backslash\{0\}\}^{\circ \circ}$ and the double inclusion

$$
B(0, r) \subset\{x: x \in C \backslash\{0\}\}^{\circ \circ} \subset B(0, R)
$$

implies that $R^{-1}\{x: x \in C \backslash\{0\}\}^{\circ \circ} \subset\left\{\|x\|^{-1} x: x \in C \backslash\{0\}\right\}^{\circ \circ}$, where it is obvious that $\left\{\|x\|^{-1} x: x \in C \backslash\{0\}\right\}^{\circ \circ}$ is a subset of $B(0,1)$. From these inclusions we get

$$
B\left(0, r R^{-1}\right) \subset\left\{\|x\|^{-1} x: x \in C \backslash\{0\}\right\}^{\circ \circ} \subset B(0,1),
$$

that states that $\left\{\|x\|^{-1} x: x \in C \backslash\{0\}\right\}$ is a norming subset of the unit sphere $S_{E}$.

From the equivalences $C^{\circ \circ} \subset B(0, R) \Leftrightarrow C \subset B(0, R)$ and

$$
B(0, r) \subset C^{\circ \circ} \subset B(0, R) \quad \Longleftrightarrow \quad B\left(0, R^{-1}\right) \subset C^{\circ} \subset B\left(0, r^{-1}\right)
$$

with $B\left(0, R^{-1}\right)=\left\{x^{*} \in E^{*}:\left\|x^{*}\right\| \leq R^{-1}\right\}$ and $B\left(0, r^{-1}\right)=\left\{x^{*} \in E^{*}:\left\|x^{*}\right\| \leq r^{-1}\right\}$, it follows that $C$ is a norming subset of $(E,\|\cdot\|)$ if and only if one of the following conditions holds: $1 . C^{\circ \circ}$ is a norming subset of $(E,\|\cdot\|) .2 . C^{\circ \circ}$ is a bounded zero neighborhood of $(E,\|\cdot\|)$. 3. $C$ and $C^{\circ}$ are bounded subsets of $(E,\|\cdot\|)$ and $\left(E^{*},\|\cdot\|\right)$, respectively. 4. $C^{\circ}$ is a norming subset of $\left(E^{*},\|\cdot\|\right)$.

If a subset $M$ of $\left(E^{*},\|\cdot\|\right)$ is uniformly bounded in a norming subset of $(E,\|\cdot\|)$ then $M$ is a bounded subset of $\left(E^{*},\|\cdot\|\right)$.

Proposition 2 (Nygaard 2001) Let $C$ be a bounded subset of a normed space $(E,\|\cdot\|)$. Then $C$ is an ubd set for $E^{*}$ if and only if $C$ is strong norming. 
Proof Let $C$ be a subset of $E$ that it is strong norming and let $M$ be a $C$-pointwise bounded subset of $\left(E^{*},\|\cdot\|\right)$. The relation

$$
C=\bigcup_{m \in \mathbb{N}}\{x \in C:|f(x)| \leq m, f \in M\}
$$

implies that there exists $C_{n}:=\{x \in C:|f(x)| \leq n, f \in M\}$ which is a norming subset of $(E,\|\cdot\|)$. As $M$ is uniformly bounded on $C_{n}$ we deduce that $M$ is a bounded subset of $\left(E^{*},\|\cdot\|\right)$, hence $C$ is an $u b d$ set for $E^{*}$.

If the bounded subset $C$ is not strong norming then $C$ is the increasing union of nonnorming sets $C_{n}, n \in \mathbb{N}$, hence there exists $f_{n} \in C_{n}^{\circ}$, with $\left\|f_{n}\right\|=n$. Since the unbounded sequence $\left(f_{n}\right)_{n}$ is $C$-pointwise bounded, we get that $C$ is not an $u b d$ set for $E^{*}$.

Following with the last part of the preceding proof, if the bounded non strong norming set $C$ is a subset of $B(0, R)$ and $C$ is the increasing union of non-norming sets $C_{n}, n \in \mathbb{N}$, then $\left\|f_{n}\right\|=n$ implies that $\left|f_{n}(x)\right| \leq R$ if $x \in n^{-1} C$. In particular, $\left|f_{n}(x)\right| \leq R$ if $x \in i^{-1} C_{i}$, for $i>n$. The relation $f_{n} \in C_{n}^{\circ}$ implies that $\left|f_{n}(x)\right| \leq 1$, if $x \in C_{n}$, and then we deduce that $\left|f_{n}(x)\right| \leq 1$ if $x \in i^{-1} C_{i}$, for $i \leq n$. Therefore the bounded set $A=\cup\left\{i^{-1} C_{i}: i \in \mathbb{N}\right\}$ is not norming for $E^{*}$, because from $\left|f_{n}(x)\right| \leq 1+R$, if $x \in A$, we deduce that $A^{\circ}$ contains the unbounded sequence $\left((1+R)^{-1} f_{n}: n \in \mathbb{N}\right)$. Additionally $\operatorname{span}\{C\}=\operatorname{span}\{A\}$. Next corollary follows directly from this observation of Proposition 2.

Corollary 1 Let $C$ be a bounded and non strong norming subset of the normed space $(E,\|\cdot\|)$. Then there exists a bounded and non-norming subset $A$ such that $\operatorname{span}\{C\}=$ $\operatorname{span}\{A\}$.

\section{A Hereditary Property of Rainwater Sets}

Let $X$ be a compact space, $C(X)$ be the space of real or complex continuous functions defined on $X$ endowed with the supremum-norm and let $\left(f_{n}: n \in \mathbb{N}\right)$ be a norm-bounded sequence in $C(X)$. From the Lebesgue dominated convergence theorem it follows that $f_{n} \rightarrow$ $f$ weakly in $C(X)$ if and only if $f_{n}(x) \rightarrow f(x)$ for every $x \in X$, that is, $\left\langle f_{n}, \mu\right\rangle \rightarrow\langle f, \mu\rangle$ for every $\mu \in C(X)^{*}$ if and only if $\left\langle f_{n}, \delta_{v}\right\rangle \rightarrow\left\langle f, \delta_{v}\right\rangle$, for each $v \in K$, where $\delta_{v}$ is the element of $S_{C(X)^{*}}$ defined by the evaluation in $v$ (see [4, IV.6.4 Corollary]). This property suggests the following definition.

Definition 11 A subset $X$ of the dual closed unit ball $B_{E^{*}}$ of a Banach space $E$ is called a Rainwater set for $E$ if every bounded sequence $\left(x_{n}\right)_{n=1}^{\infty}$ of $E$ that converges pointwise on $X$, i.e., such that $x^{*} x_{n} \rightarrow x^{*} x$ for each $x^{*} \in X$, converges weakly in $E$, i.e., $x^{*} x_{n} \rightarrow x^{*} x$ for each $x^{*} \in E^{*}$ (cf. [6]).

Then for a compact space $X$ we have that the set $\left\{\delta_{v}: v \in X\right\}$ is a Rainwater set for $C(X)$.

Rainwater's classic theorem [19] asserts that the set of extreme points of the closed dual unit ball of a Banach space $E$ is a Rainwater set for $E$. According to [21, Corollary 11], each James boundary of $E$ is a Rainwater set for $E$. As regards the Banach space $C(X)$ of real-valued continuous functions over a compact space $X$ equipped with the supremum norm, the Arens-Kelly theorem asserts that the set of the extreme points of the compact 
subset $B_{C(X)^{*}}$ of $C(X)^{*}$ (weak $^{*}$ ) is $\left\{ \pm \delta_{x}: x \in X\right\}$ (see [1]). Then we get again Rainwater's theorem for real $C(X)$, stating that $\left\{\delta_{x}: x \in X\right\}$ is a Rainwater set for $C(X)$.

Note that a sequence $\left(x_{n}\right)_{n=1}^{\infty}$ of $E$ converges pointwise on a subset $X$ of $E^{*}$ if and only if it converges pointwise in $\operatorname{span}\{X\}$, therefore the condition $X \subset B_{E^{*}}$ may be avoided in the definition of Rainwater set.

The fact that a normed space $E$ lives isometrically in $E^{* *}$ suggests the first part of the following definition.

Definition 12 Let $M$ be a subset of a normed space $E . M$ is a Rainwater set for $E^{*}$ if every bounded sequence $\left(x_{n}^{*}: n \in \mathbb{N}\right)$ of $E^{*}$ that converges pointwise on $M$, i.e., such that $x x_{n}^{*} \rightarrow x x^{*}$ for each $x \in M$, converges weakly in $E^{*}$, i.e., $x^{* *} x_{n}^{*} \rightarrow x^{* *} x^{*}$ for each $x^{* *} \in E^{* *}$.

$M$ is a strong [web] Rainwater set for $E^{*}$ if for each increasing covering $\left(M_{n}: n \in \mathbb{N}\right)$ [web $\left(M_{\sigma}: \sigma \in \mathbb{N}^{<\infty}\right)$ ] of $M$ there exists $n_{0} \in \mathbb{N}$ [a chain $\left(\sigma_{n}: n \in \mathbb{N}\right)$ in $\mathbb{N}^{<\infty}$ ] for which $M_{n_{0}}$ [each $M_{\sigma_{n}}, n \in \mathbb{N}$ ], is a Rainwater set for $E^{*}$.

Proposition 3 Let $M$ be a subset of the normed space $E$ that it is both an ubd and a Rainwater set for $E^{*}$. Then each sequence $\left(x_{n}^{*}: n \in \mathbb{N}\right)$ of $E^{*}$ that pointwise converges on $M$ converges weakly.

In particular, if $\mathcal{M}$ is a subfamily of an algebra of sets $\mathcal{A}$ such that $\mathcal{M}$ is a Nikodým set for ba $(\mathcal{A})$ and $\left\{\chi_{A}: A \in \mathcal{M}\right\}$ is a Rainwater set for ba $(\mathcal{A})$, then $\mathcal{M}$ is a Grothendieck set for $b a(\mathcal{A})$.

Proof As $M$ is an $u b d$ set for $E^{*}$ and $\left(x_{n}^{*}: n \in \mathbb{N}\right)$ pointwise converges on $M$ then $\left(x_{n}^{*}\right.$ : $n \in \mathbb{N})$ is a bounded sequence. The proposition follows from the Rainwater condition.

Let $\left(\mu_{n}: n \in \mathbb{N}\right)$ be a sequence in $b a(\mathcal{A})$ such that $\mu_{n}(A)=\left\langle\chi_{A}, \mu_{n}\right\rangle \rightarrow \mu(A)=$ $\left\langle\chi_{A}, \mu\right\rangle$, for every $A \in \mathcal{M}$. Applying the general case with $M=\left\{\chi_{A}: A \in \mathcal{M}\right\}, E=$ $\ell_{0}^{\infty}(\mathcal{A}), E^{*}=b a(\mathcal{A})$ and $x_{n}^{*}=\mu_{n}, n \in \mathbb{N}$, we get that $\left(\mu_{n}: n \in \mathbb{N}\right)$ converges weakly to $\mu$, hence $\mathcal{M}$ is a Grothendieck set for $b a(\mathcal{A})$.

With the hypothesis of Proposition 3 the next theorem states that $M$ and $\left\{\chi_{A}: A \in \mathcal{M}\right\}$ are strong Rainwater sets for $E^{*}$ and $b a(\mathcal{A})$, respectively. Its argument is based in the proof of [8, Theorem 8].

Theorem 2 Let $M$ be a subset of a normed space $E$ that it is both an ubd and a Rainwater set for $E^{*}$. Then $M$ is a strong Rainwater set for $E^{*}$.

In particular, if $\mathcal{M}$ is a subfamily of an algebra of sets $\mathcal{A}$ such that $\mathcal{M}$ is a Nikodým set for ba $(\mathcal{A})$ and $\left\{\chi_{A}: A \in \mathcal{M}\right\}$ is a Rainwater set for ba $(\mathcal{A})$ then $\left\{\chi_{A}: A \in \mathcal{M}\right\}$ is a strong Rainwater set for ba $(\mathcal{A})$.

Proof We may suppose that $M \subset B_{E}$. Let $\left\{M_{n}: n \in \mathbb{N}\right\}$ be an increasing covering of $M$ and let $\widehat{E}$ be the completion of $E$.

First we claim that $\widehat{E}=\cup_{n \in \mathbb{N}} n \overline{\mathrm{abx} M_{n}} \widehat{E}$ and we prove this equality by contradiction. If there exists $x \in S_{\widehat{E}}$ such that $x \notin n \overline{\mathrm{abx} M_{n}} \widehat{E}$, for all $n \in \mathbb{N}$, then the separation theorem provides $x_{n}^{*} \in E^{*}$ with $\left|x_{n}^{*}(x)\right|=1$ and

$$
\sup \left\{\left|x_{n}^{*}(y)\right|: y \in{\overline{\operatorname{abx} M_{n}}}^{\widehat{E}}\right\} \leq \frac{1}{n} .
$$


If $y \in M$ there exists $k \in \mathbb{N}$ such that $y \in M_{n}$ for every $n \geq k$. Consequently

$$
\left|x_{n}^{*}(y)\right| \leq \frac{1}{n} \quad \text { for } n \geq k,
$$

which shows that $x_{n}^{*}(y) \rightarrow 0$, hence as $M$ is an $u b d$ set for $E^{*}$ the sequence $\left(x_{n}^{*}: n \in\right.$ $\mathbb{N})$ is bounded in $E^{*}$. As $M$ is a Rainwater for $E^{*}$ and the bounded sequence $\left(x_{n}^{*}: n \in\right.$ $\mathbb{N}$ ) converges to 0 pointwise in $M$ then $x_{n}^{*} \rightarrow 0$ weakly in $E^{*}$. This is a contradiction, since $\left|x_{n}^{*}(x)\right|=1$ for every $n \in \mathbb{N}$. The claim is proved and the relations $M \subset B_{E}$ and $\widehat{E}=\cup_{n \in \mathbb{N}} n{\overline{\text { abx } M_{n}}}^{\widehat{E}}$ implies that there exists $p \in \mathbb{N}$ such that the unit closed ball $B_{\widehat{E}}$ of $\widehat{E}$ verifies that

$$
M \subset B_{\widehat{E}} \subset p{\overline{\operatorname{abx} M_{p}}}^{\widehat{E}},
$$

and it follows that $\overline{\operatorname{abx} M}_{p} \widehat{E}$ is a Rainwater set for $E^{*}$. In order to prove this theorem it suffices to show that $\operatorname{abx} M_{p}$ is also a Rainwater set for $E^{*}$, because then $M_{p}$ shall be a Rainwater set for $E^{*}$.

Let $\left\{y_{n}^{*}\right\}_{n=1}^{\infty}$ be a bounded subset of $E^{*}$ such that $y_{n}^{*}(x) \rightarrow 0$ for each $x \in \operatorname{abx} M_{p}$ and let $\epsilon>0$. For each $z \in \overline{\operatorname{abx} M_{p}} \widehat{E}$ there exists $x_{\epsilon, z} \in \operatorname{abx} M_{p}$ such that

$$
\left|y_{n}^{*}\left(z-x_{\epsilon, z}\right)\right|<\frac{\epsilon}{2} \quad \text { for each } n \in \mathbb{N} .
$$

Since $y_{n}^{*}\left(x_{\epsilon, z}\right) \rightarrow 0$, it follows that there exists $n_{\epsilon, z}$ such that

$$
\left|y_{n}^{*}\left(x_{\epsilon, z}\right)\right|<\frac{\epsilon}{2} \quad \text { for each } n>n_{\epsilon, z} .
$$

From (1) and (2) it follows that $\left|y_{n}^{*}(z)\right|<\epsilon$ if $n>n_{\epsilon, z}$, hence the equality $y_{n}^{*}(z) \rightarrow 0$, for each $z \in{\overline{\operatorname{abx} M_{p}}}^{\widehat{E}}$, implies that $\mu\left(y_{n}^{*}\right) \rightarrow 0$, for each $\mu \in E^{* *}$, hence abx $M_{p}$ is a Rainwater set for $E^{*}$.

The particular case follows from Lemma 1 and the result just proved with $E=\ell_{0}^{\infty}(\mathcal{A})$ and $M=\left\{\chi_{A}: A \in \mathcal{M}\right\}$.

Corollary 2 Let $M$ be a subset of a Banach space $E$ that it is both a strong ubd and a Rainwater set for $E^{*}$. Then for each increasing covering $\left(M_{n}: n \in \mathbb{N}\right)$ of $M$ there exists $p \in \mathbb{N}$ such that $M_{q}$, for each $q \geq p$, is both an ubd and Rainwater set for $E^{*}$. If additionally $M$ is a web ubd set for $E^{*}$ then for each web $\left(M_{\sigma}: \sigma \in \mathbb{N}<\infty\right)$ in $M$ there exists a chain $\left(\sigma_{n}: n \in \mathbb{N}\right)$ in $\mathbb{N}^{<\infty}$ such that each $M_{\sigma_{q}}$, for $q \in \mathbb{N}$, is both an ubd and Rainwater set for $E^{*}$. Hence, for each sequence $\left(x_{n}^{*}: n \in \mathbb{N}\right)$ of $E^{*}$ the pointwise convergence in one of these $M_{q}, q \geq p$, or $M_{\sigma_{q}}, q \in \mathbb{N}$, respectively, implies the weak convergence of $\left(x_{n}^{*}: n \in \mathbb{N}\right)$.

Proof The strong $u b d$ set $M$ is by Theorem 2 a strong Rainwater set for $E^{*}$, hence if $\left(M_{n}: n \in \mathbb{N}\right)$ is an increasing covering of $M$ there exists $p \in \mathbb{N}$ such that $M_{q}$ is both an $u b d$ and a Rainwater set for $E^{*}$ for each $q \geq p$.

The second part follows by induction, taking care that if $M$ is a web $u b d$ set for $E^{*}$ and $\left(M_{\sigma}: \sigma \in \mathbb{N}^{<\infty}\right)$ is a web in $M$ then there exists $p_{1}$ such that for every $q_{1} \geq p_{1}$ the set $M_{q_{1}}$ is also web $u b d$ for $E^{*}$. The final part follows from Proposition 3.

Corollary 3 Let $\mathcal{M}$ be a subfamily of an algebra of sets $\mathcal{A}$ such that $\mathcal{M}$ is both a strong Nikodým and Grothendieck set for ba $(\mathcal{A})$. Then for each increasing covering $\left(\mathcal{M}_{n}: n \in \mathbb{N}\right)$ of $\mathcal{M}$ there exists $p \in \mathbb{N}$ such that, for each $q \geq p, \mathcal{M}_{q}$ is both a Nikodým set and a 
Grothendieck set for $b a(\mathcal{A})$. If additionally $\mathcal{M}$ is a web Nikodým set for ba $(\mathcal{A})$ then for each web $\left(\mathcal{M}_{\sigma}: \sigma \in \mathbb{N}<\infty\right)$ in $\mathcal{M}$ there exists a chain $\left(\sigma_{n}: n \in \mathbb{N}\right)$ in $\mathbb{N}^{<\infty}$ such that $\mathcal{M}_{\sigma_{q}}$, for each $q \in \mathbb{N}$, is both a Nikodým and Grothendieck set for ba $(\mathcal{A})$.

Hence if $\mathcal{M}$ is a subfamily of an algebra of sets $\mathcal{A}$ such that $\mathcal{M}$ is a strong Nikodým (web Nikodým) set for ba $(\mathcal{A})$ and $\left\{\chi_{M}: M \in \mathcal{M}\right\}$ is Rainwater set for ba $(\mathcal{A})$ then $\mathcal{M}$ is a strong Grothendieck (web Grothendieck) set for $b a(\mathcal{A})$.

Proof Let $\mathcal{M}$ be both a strong Nikodým and Grothendieck set for $b a(\mathcal{A})$. Then $\left\{\chi_{M}: M \in\right.$ $\mathcal{M}$ \} is a Rainwater set for $b a(\mathcal{A})$. Let $\left(\mathcal{M}_{n}: n \in \mathbb{N}\right)$ be an increasing covering of $\mathcal{M}$. Applying Lemma 1 and Corollary 2 with $E=\ell_{0}^{\infty}(\mathcal{A})$ and $M=\left\{\chi_{A}: A \in \mathcal{M}\right\}$ we get that there exists $p \in \mathbb{N}$ such that, for each $q \geq p$, the set $\left\{\chi_{A}: A \in \mathcal{M}_{q}\right\}$ is both an $u b d$ set and a Rainwater set for $b a(\mathcal{A})$. By Lemma 1 and the final statement of Proposition 3 we get that $\mathcal{M}_{q}$ is both a Nikodým set and a Grothendieck set for $b a(\mathcal{A})$. Hence, $\mathcal{M}$ is a strong Grothendieck set for $b a(\mathcal{A})$.

With obvious changes we get the corresponding proof for the web Nikodým case.

\section{Application to Bounded Measures and Open Problems}

Next lemma is a particular case of [8, Theorem 6], that is based on [7, Proposition 14]. We provide a brief proof for the sake of completeness.

Lemma 2 Let $\Sigma$ be a $\sigma$-algebra of sets. Then $\Sigma$ is a Grothendieck set for ba $(\Sigma)$.

Proof Let $\left(\mu_{n}: n \in \mathbb{N}\right)$ be a sequence in $b a(\Sigma)$ such that $\mu_{n}(A) \rightarrow \mu(A)$ for each $A \in \mathcal{A}$. As $\Sigma$ is a Nikodým set for $b a(\Sigma)$ we have that the set $M=\left\{\mu_{n}: n \in \mathbb{N}\right\}$ is a bounded subset of $b a(\mathcal{A})$. Since the $\sigma$-algebra $\Sigma$ has property $(G)$ then by Theorem 1, [3, Corollary 5.2] and $[4,4.9 .12$ Theorem] we get that the bounded set $M$ is a relatively weakly sequentially compact and then, from $\mu_{n}(A) \rightarrow \mu(A)$ for each $A \in \mathcal{A}$, we deduce that $\mu$ is the only possible weakly adherent point of the sequence $\left(\mu_{n}: n \in \mathbb{N}\right)$. Hence, $\mu_{n} \rightarrow \mu$ weakly and we get that $\Sigma$ is a Grothendieck set for $b a(\Sigma)$.

In the next three results we improve the strong Grothendieck properties considered in [8, Theorem 3, Corollary 10, and Proposition 12] to the corresponding web Grothendieck properties.

Theorem 3 Each $\sigma$-algebra $\Sigma$ of subsets of a set $\Omega$ is a web Grothendieck set for ba $(\Sigma)$. More precisely, if $\left(\Sigma_{\sigma}: \sigma \in \mathbb{N}<\infty\right)$ is a web in $\Sigma$ there exists a chain $\left(\sigma_{n}: n \in \mathbb{N}\right)$ in $\mathbb{N}<\infty$ such that each $\Sigma_{\sigma_{n}}, n \in \mathbb{N}$, is both a Nikodým and a Grothendieck set for ba $(\Sigma)$.

Proof It is well known that each $\sigma$-algebra $\Sigma$ of subsets of a set $\Omega$ has web Nikodým property (see [15] or [13]) and $(G)$ property. Then Lemma 2 implies that $\Sigma$ is a Grothendieck set for $b a(\Sigma)$. The conclusion follows from Corollary 3 with $\mathcal{M}=\Sigma$.

Corollary 4 If $\left(\Lambda_{\sigma}: \sigma \in \mathbb{N}<\infty\right)$ is a web in $2^{\mathbb{N}}$, there exists a chain $\left(\sigma_{n}: n \in \mathbb{N}\right)$ in $\mathbb{N}<\infty$ such that each $\Lambda_{\sigma_{n}}, n \in \mathbb{N}$, is both a Nikodým and a Grothendieck set for ba $\left(2^{\mathbb{N}}\right)=\ell_{\infty}^{*}$. 
Proof Apply Theorem 3 to the $\sigma$-algebra $2^{\mathbb{N}}$.

Theorem 3 allows to extend various classic theorems of Banach space theory. As a sample, we extend the classical Phillips lemma about convergence in $b a(\Sigma)$.

Proposition 4 Let $\left(\Sigma_{\sigma}: \sigma \in \mathbb{N}^{<\infty}\right)$ be a web in a $\sigma$-algebra $\Sigma$ of subsets of a set $\Omega$. There exists a chain $\left(\sigma_{n}: n \in \mathbb{N}\right)$ in $\mathbb{N}<\infty$ such that each $\Sigma_{\sigma_{p}}, p \in \mathbb{N}$, verifies that if $(\mu)_{n n=1}^{\infty} \subseteq$ ba $(\Sigma)$ satisfies that $\lim _{n \rightarrow \infty} \mu_{n}(A)=0$ for every $A \in \Sigma_{\sigma_{p}}$ and $\left(A_{k}: k \in \mathbb{N}\right)$ is a sequence of pairwise disjoint elements of $\Sigma$, then

$$
\lim _{n \rightarrow \infty} \sum_{k=1}^{\infty}\left|\mu_{n}\left(A_{k}\right)\right|=0 .
$$

Proof According to Theorem 3 there exists a chain $\left(\sigma_{n}: n \in \mathbb{N}\right)$ in $\mathbb{N}<\infty$ such that each $\Sigma_{\sigma_{p}}, p \in \mathbb{N}$, is Grothendieck set for $b a(\Sigma)$. So, if $\lim _{n \rightarrow \infty} \mu_{n}(A)=0$ for every $A \in \Sigma_{p}$, then $\mu_{n} \rightarrow \mu$ weakly in $b a(\Sigma)$. In particular, $\mu_{n}(A) \rightarrow \mu(A)$ for every $A \in \Sigma$. Hence, (3) holds by Phillip's classic theorem.

We do not know the answer of the following two questions for an algebra $\mathcal{A}$ of subsets of a set $\Omega$ :

Question 1 Let us suppose that $\mathcal{A}$ is a Grothendieck set for $b a(\mathcal{A})$. Is $\mathcal{A}$ a strong Grothendieck set for $b a(\mathcal{A})$ ?

Question 2 Does the Nikodým property of $\mathcal{A}$ imply that $\mathcal{A}$ has the strong Nikodým property for $b a(\mathcal{A})$ ? This open question was proposed in 2013 by Valdivia in [25].

Acknowledgements The second author is supported by Grant PGC2018-094431-B-I00 of the Ministry of Science, Innovation and Universities of Spain.

We thank the reviewers for their useful comments and suggestions.

We also thank to Professor Juan Carlos Ferrando for careful reading the manuscript and valuable discussions and to Professor José Mas for his assistance with LaTex.

\section{References}

1. Arens, R.F., Kelley, J.L.: Characterizations of the space of continuous functions over a compact Hausdorff space. Trans. Am. Math. Soc. 62, 499-508 (1947)

2. Diestel, J., Faires, B., Huff, R.: Convergence and boundedness of measures in non $\sigma$-complete algebras. Preprint (1976)

3. Diestel, J., Uhl, J.J.: Vector Measures. Mathematical Surveys and Monographs, vol. 15. American Mathematical Society, Providence (1977)

4. Dunford, N., Schwartz, J.T.: Linear Operators. Part I: General Theory. Wiley, New Jersey (1988)

5. Fernández, J., Hui, S., Shapiro, H.: Unimodular functions and uniform boundedness. Publ. Mat. 33, 139-146 (1989)

6. Ferrando, J.C., Ka̧kol, J., López-Pellicer, M.: On spaces $C^{b}(X)$ weakly $K$-analytic. Math. Nachr. 290, 2612-2618 (2017)

7. Ferrando, J.C., López-Alfonso, S., López-Pellicer, M.: On Nikodým and Rainwater sets for $b a(\mathcal{R})$ and a problem of M. Valdivia. Filomat 33, 2409-2416 (2019)

8. Ferrando, J.C., López-Alfonso, S., López-Pellicer, M.: On the Grothendieck property (submited) (2020)

9. Ferrando, J.C., López-Pellicer, M., Sánchez Ruiz, L.M.: Metrizable Barrelled Spaces. Pitman Research Notes in Mathematics Series, vol. 332. Longman, Harlow (1995) 
10. Ferrando, J.C., Sánchez Ruiz, L.M.: A survey on recent advances on the Nikodým boundedness theorem and spaces of simple functions. Rocky Mount. J. Math. 34, 139-172 (2004)

11. Fonf, V.P.: On exposed and smooth points of convex bodies in Banach spaces. Bull. Lond. Math. Soc. 28, 51-58 (1996)

12. Kąkol, J., López-Pellicer, M.: On Valdivia strong version of Nikodým boundedness property. J. Math. Anal. Appl. 446, 1-17 (2017)

13. López-Alfonso, S., Mas, J., Moll, S.: Nikodým boundedness property and webs in $\sigma$-algebras. Rev. R. Acad. Cienc. Exactas Fís. Nat. Ser. A Math. RACSAM 110, 711-722 (2016)

14. López-Alfonso, S.: On Schachermayer and Valdivia results in algebras of Jordan measurable sets. Rev. R. Acad. Cienc. Exactas Fís. Nat. Ser. A Mat. RACSAM 110, 799-808 (2016)

15. López-Pellicer, M.: Webs and bounded finitely additive measures. J. Math. Anal. Appl. 210, 257-267 (1997)

16. Nygaard, O.: A strong uniform boundedness principle in Banach spaces. Proc. Am. Math. Soc. 129, 861-863 (2001)

17. Nygaard, O.: Thick sets in Banach spaces and their properties. Quaest. Math. 29, 50-72 (2006)

18. Plebanek, G., Sobota, D.: Countable tightness in the spaces of regular probability measures. Fund. Math. 229, 159-170 (2015)

19. Rainwater, J.: Short notes: Weak convergence of bounded sequences. Proc. Am. Math. Soc. 14, 999-999 (1963)

20. Schachermayer, W.: On some classical measure-theoretic theorems for non-sigma-complete Boolean algebras. Diss. Math. (Rozprawy Mat.) 214, 1-33 (1982)

21. Simons, S.: A convergence theorem with boundary. Pac. J. Math. 40, 703-708 (1972)

22. Sobota, D., Zdomskyy, L.: The Nikodým property in the Sacks model. Topol. Appl. 230, 24-34 (2017)

23. Talagrand, M.: Propriété de Nikodým and propriété de Grothendieck. Stud. Math. 78, 165-171 (1984)

24. Valdivia, M.: On certain barrelled normed spaces. Ann. Inst. Fourier 29, 39-56 (1979)

25. Valdivia, M.: On Nikodým boundedness property. Rev. R. Acad. Cienc. Exactas Fís. Nat. Ser. A Mat. RACSAM 107, 355-372 (2013)

Publisher's Note Springer Nature remains neutral with regard to jurisdictional claims in published maps and institutional affiliations. 